\title{
Performance Analysis of Coded Communication Systems on Nakagami Fading Channels With Selection Combining Diversity
}

\author{
Ramesh Annavajjala, Student Member, IEEE, A. Chockalingam, Senior Member, IEEE, and
} Laurence B. Milstein, Fellow, IEEE

\begin{abstract}
In this paper, we develop analytical tools for the performance analysis of coded, coherent communication systems on independent and identically distributed Nakagami- $m$ fading channels with selection combining (SC) diversity. First, we derive an exact expression for the moment generation function (MGF) of the signal-to-noise ratio (SNR) of a code symbol at the output of the selection combiner. Next, based on Gauss-Chebyshev quadrature and Gauss-Laguerre quadrature rules, we propose a simple to compute, yet accurate, numerical solution for the pairwise error probability (PEP) of coded $M$-phase-shift keying (PSK) signals. Using the PEP expressions, we present the union bound-based biterror performance of trellis-coded modulation schemes and turbo codes. Finally, we derive an exact expression for the computational cutoff rate of a coded system with $M$-PSK signaling and SC diversity, and show that the cutoff rate expression is a simple function of the MGF of the SNR at the output of the diversity combiner.
\end{abstract}

Index Terms-Coded systems, cutoff rate, Nakagami fading, selection combining (SC), trellis-coded modulation (TCM), turbo codes.

\section{INTRODUCTION}

D IVERSITY reception, together with channel coding, is a popular technique to mitigate the deleterious effects of multipath fading in a mobile radio environment [1], [2]. Trelliscoded modulation (TCM) schemes are known to provide good coding gains without incurring bandwidth expansion, and are considered for bandwidth-limited wireline communication systems and bandwidth- and power-limited wireless communication systems [3], [4], whereas turbo codes are popular in coding theory literature for their near-Shannon-limit error performance [5]. Indeed, turbo codes have been proposed for third-generation wireless communication standards [6]. Union bounding techniques are usually employed to obtain upper bounds on the error probability of coded systems, but one of the difficulties with this approach lies in obtaining a closed-form expression for the pairwise error probability (PEP) between two codewords. Often, for simplicity, a Chernoff-bound-type PEP expression is used, re-

Paper approved by P. Y. Kam, the Editor for Modulation and Detection for Wireless Systems of the IEEE Communications Society. Manuscript received December 20, 2002; revised July 10, 2003 and December 23, 2003. This work was supported in part by the Office of Naval Research under Grant N00014-02-1-0001, and in part by the TRW Foundation.

R. Annavajjala and L. B. Milstein are with the Department of Electrical and Computer Engineering, University of California at San Diego, La Jolla, CA 92093-0407 USA (e-mail: ramesh@cwc.ucsd.edu; milstein@ece.ucsd.edu).

A. Chockalingam is with the Department of Electrical Communication Engineering, Indian Institute of Science, Bangalore 560012, India (e-mail: achockal@ece.iisc.ernet.in).

Digital Object Identifier 10.1109/TCOMM.2004.831386 sulting in a loss of tightness by about $4 \mathrm{~dB}$ in the union bound on the error probability on fading channels [4], [7]-[9]. For simple channel models, like the additive white Gaussian noise (AWGN) and the Rayleigh fading channels, one can obtain the PEP in closed form [10], [11]. Recently, for Ricean fading and log-normal shadowing channels, Tellambura [12] has obtained a numerical approximation for the PEP using the Gauss-Chebyshev quadrature (GCQ) rule.

Knowledge of the channel cutoff rate [13] is very valuable in assessing the performance of practical coding schemes. Al-Semari and Fuja [14] derived both the cutoff rate and the union bound on the error probability using the Chernoff bound on the PEP for various diversity schemes on Rayleigh fading channels. In this paper, we are concerned with the performance, as measured by the channel cutoff rate, as well as the error performance of practical coding schemes, like TCM and turbo codes, on Nakagami fading channels with selection combining (SC) diversity. In particular, we obtain an exact expression for the moment generating function (MGF) of the signal-to-noise ratio (SNR) for an $M$-phase-shift keying (PSK) code symbol at the output of the selection combiner. Next, using GCQ and Gauss-Laguerre quadrature (GLQ) rules, we provide a simple to compute, yet accurate, numerical solution for the PEP with $M$-PSK modulated codewords on independent and identically distributed (i.i.d.) Nakagami- $m$ fading channels with $L$-antenna SC diversity. Using the union bounding technique, we present the bit-error probability (BEP) performance of TCM and turbo codes. We also derive an exact expression for the cutoff rate of coded communication systems on i.i.d. Nakagami- $m$ fading with $L$-antenna SC diversity, and show that it is a simple function of the MGF of the diversity combiner's output SNR.

The rest of this paper is organized as follows. In Section II, we present the system model. An exact expression for the MGF of the code symbol SNR, as well as a simple GCQ/GLQ-based expression for the PEP with SC diversity, is presented in Section III. An exact expression for the cutoff rate with $L$-path SC diversity is derived in Section IV. In Section V, we present the union-bound-based error-probability performance of both TCM and turbo codes. Finally, we conclude this paper in Section VI.

\section{SYSTEM MODEL}

We assume that the transmitted code symbols are $M$-PSK modulated, and are coherently demodulated at the receiver. The receiver employs $L$ antennas for diversity combining to miti- 
gate the effect of multipath fading. Let $\mathbf{X}=\left(X_{1}, \ldots, X_{N}\right)$ denote the transmitted code symbol sequence. Then the received symbol sequence on the $l$ th antenna, $r_{k}^{(l)}$, is given by

$$
\begin{aligned}
r_{k}^{(l)} & =\sqrt{E_{s}} \alpha_{k}^{(l)} e^{j \theta_{k}^{(l)}} X_{k}+\eta_{k}^{(l)}, \\
k & =1,2, \ldots, N ; \quad l=1,2, \ldots, L
\end{aligned}
$$

where $\alpha_{k}^{(l)}$ is the fade random variable, and $\eta_{k}^{(l)}$ is the AWGN component associated with the $l$ th antenna path when the transmitted data symbol $X_{k} \in\left\{e^{j 2 \pi p / M}\right\}_{p=0}^{M-1}$. The code symbols $X_{k}$ are assumed to have unit energy, the $\eta_{k}^{(l)}$ are assumed to be i.i.d. complex Gaussian with zero mean and variance $\sigma^{2}=N_{0}$, where $N_{0} / 2$ is the two-sided power spectral density of the underlying noise random process $\eta(t)$, and $E_{s}$ is the energy-per-code symbol-per-branch. Further, the fade random variables, $\alpha_{k}^{(l)}$, are assumed to be i.i.d. and are Nakagami- $m$ distributed with the probability density function (pdf) and the cumulative distribution function (cdf), respectively, given by

$$
\begin{aligned}
& f_{\alpha}(x)=\frac{2 m^{m}}{\Gamma(m)} e^{-m x^{2}} x^{2 m-1}, \quad x \geq 0 \\
& F_{\alpha}(x)=\gamma\left(m, m x^{2}\right), \quad x \geq 0
\end{aligned}
$$

where $\Gamma(m)$ is the standard Gamma integral and $\gamma(a, x)=$ $\int_{u=0}^{x} e^{-u} u^{a-1} d u$ is the incomplete Gamma function [15]. Here, we normalized the second moment of the fade, $E\left(\alpha^{2}\right)$, to unity, and $m \geq 1 / 2$. The random phase, $\theta_{k}^{(l)}$, associated with the $k$ th code symbol on the $l$ th antenna, is uniformly distributed in $(-\pi, \pi)$. Both $\alpha_{k}^{(l)}$ and $\theta_{k}^{(l)}$ are assumed to remain constant over one code symbol duration. In the following section, we obtain expressions for the MGF and the PEP with $L$-path SC diversity.

\section{MGF AND PEP WITH SC DIVERSITY}

The PEP with SC diversity, $P^{\mathrm{SC}}(\mathbf{X} \rightarrow \mathbf{Y})$, is the probability that the transmitted codeword $\mathbf{X}=\left(X_{1}, \ldots, X_{N}\right)$ is incorrectly decoded as $\mathbf{Y}=\left(Y_{1}, \ldots, Y_{N}\right)$. If the two codewords $\mathbf{X}$ and $\mathbf{Y}$ differ in $d$ positions, ${ }^{1}$ then the PEP is denoted by $P^{\mathrm{SC}}{ }_{2}(d)$, which we derive as follows.

The selection combiner picks the received signal for coherent combining from the path on which the instantaneous fade amplitude $\alpha_{k}^{(n)}$ is maximum. Accordingly, from (1), the decision statistic $r_{k}$, at the output of the selection combiner, is given by $r_{k}=r_{k}^{(n)} \alpha_{k}^{(n)} e^{-j \theta_{k}^{(n)}}$, where $\alpha_{k}^{(n)}=\max \left(\alpha_{k}^{(1)}, \ldots, \alpha_{k}^{(L)}\right)$. By defining

$$
\beta_{k}=\left[\tilde{\alpha}_{k}\right]^{2}=\max \left(\left[\alpha_{k}^{(1)}\right]^{2}, \ldots,\left[\alpha_{k}^{(L)}\right]^{2}\right)
$$

we can simplify $r_{k}$ as

$$
r_{k}=\beta_{k} \sqrt{E_{s}} X_{k}+\eta_{k}
$$

where $\eta_{k}$ is easily shown to be a conditional zero-mean complex Gaussian random variable with conditional variance $\beta_{k} N_{0}$. With this statistic at the output of the demodulator, the

${ }^{1}$ Without loss of generality, we assume that codewords $\mathbf{X}$ and $\mathbf{Y}$ differ in the first $d$ positions. conditional PEP $P_{2}^{\mathrm{SC}}\left(d \mid \beta_{1}, \ldots, \beta_{d}\right)$, conditioned on the fading random variables $\beta_{1}, \beta_{2}, \ldots, \beta_{d}$, is given by [16]

$$
P_{2}^{\mathrm{SC}}\left(d \mid \beta_{1}, \ldots, \beta_{d}\right)=Q\left(\sqrt{\sum_{n=1}^{d} a_{n} \beta_{n}}\right)
$$

where $a_{n}=\left(E_{s}\left|X_{n}-Y_{n}\right|^{2}\right) /\left(2 N_{0}\right)$.

The unconditional PEP $P^{\mathrm{SC}_{2}}(d)$ is obtained by averaging the above expression over the distributions of $\beta_{1}, \beta_{2}, \ldots, \beta_{d}$, and is given by

$$
\begin{aligned}
P_{2}^{\mathrm{SC}}(d) & =E_{\beta_{1}, \beta_{2}, \ldots, \beta_{d}}\left[Q\left(\sqrt{\sum_{n=1}^{d} a_{n} \beta_{n}}\right)\right] \\
& =\frac{1}{\pi} \int_{\phi=0}^{\frac{\pi}{2}} d \phi \prod_{n=1}^{d} E_{\beta_{n}}\left[e^{-\frac{a_{n} \beta_{n}}{2 \sin ^{2} \phi}}\right] \\
& =\frac{1}{\pi} \int_{\phi=0}^{\frac{\pi}{2}} d \phi \prod_{n=1}^{d} \operatorname{MGF}_{\beta_{n}}\left(-\frac{a_{n}}{2 \sin ^{2} \phi}\right)
\end{aligned}
$$

where we have used the identity $Q(x)=(1 / \pi) \int_{\phi=0}^{(\pi / 2)}$ $e^{-\left(x^{2}\right) /\left(2 \sin ^{2} \phi\right)} d \phi$ for $x \geq 0$ from [17], and the independence among the random variables $\beta_{1}, \ldots, \beta_{d}$. The last step in (7) is due to the definition of the MGF of the random variable $Z$, defined as $\operatorname{MGF}_{Z}(s)=E_{Z}\left[e^{s Z}\right]$.

\section{A. An Exact Expression for the MGF}

Using (2), (3), and (4), we can directly obtain the pdf of $\beta_{k}$ as

$$
f_{\beta_{k}}(x)=\frac{L m^{m}}{\Gamma(m)} e^{-m x} x^{m-1}[\gamma(m, m x)]^{L-1}, \quad x \geq 0
$$

and the MGF of $\beta_{k}$ can be expressed as

$\operatorname{MGF}_{\beta_{k}}(s)=\frac{L m^{m}}{\Gamma(m)} \int_{x=0}^{\infty} e^{-x(m-s)} x^{m-1}[\gamma(m, m x)]^{L-1} d x$.

In order to simplify the above equation, we express $\gamma(m, m x)$ of (9) in terms of the confluent hypergeometric function ${ }_{1} F_{1}(\cdot ; \cdot ; \cdot)$ using the relationship $\gamma(n, x)=$ $(1 / n) e^{-x} x^{n}{ }_{1} F_{1}(1 ; 1+n ; x)[15$, eq. (8.351.2)]. With this, a closed-form solution for (9) can be obtained as

$$
\begin{aligned}
\operatorname{MGF}_{\beta_{k}}(s)= & \frac{L m^{L(m-1)+1}}{\Gamma(m)} \int_{x=0}^{\infty} e^{-x(m L-s)} x^{m L-1} \\
& \times\left[{ }_{1} F_{1}(1 ; 1+m ; m x)\right]^{L-1} d x \\
= & \frac{L m^{L(m-1)+1} \Gamma(m L)}{\Gamma(m)} \frac{1}{(m L-s)^{m L}} \\
& \times F_{A}(m L ; \underbrace{1, \ldots, 1}_{L-1 \text { times }} ; \underbrace{1+m, \ldots, 1+m}_{L-1 \text { times }} ; \\
& \underbrace{\frac{m}{m L-s}, \ldots, \frac{m}{m L-s}}_{L-1 \text { times }})
\end{aligned}
$$


where the simplification is due to $[15$, eq. (9.19)] and $F_{A}(\cdot ; \cdots ; \cdots ; \cdots)$ is Laurecella's hypergeometric function $[15$, eq. (9.19)].

\section{B. A Simple and Accurate Numerical Solution}

Direct substitution of (10) in (7) does not seem to result in a closed-form expression for $P_{2}^{\mathrm{SC}}(d)$. Also, for large values of $L$, a numerical approximation to $\mathrm{MGF}_{\beta_{n}}(s)$ is simpler to implement than the direct evaluation using (10). With this motivation, we develop accurate numerical approximations for both $\mathrm{MGF}_{\beta_{n}}(s)$ and $P_{2}^{\mathrm{SC}}(d)$.

According to the GLQ rule, we have [18]

$$
\int_{x=0}^{\infty} e^{-x} x^{Q} f(x) d x \approx \sum_{j=1}^{J} w_{Q}(j) f\left(x_{Q}(j)\right)
$$

where $\left\{w_{Q}(j)\right\}$ are the weights of the GLQ integration rule for a specific $Q$ [18]. Using this to approximate $\operatorname{MGF}_{\beta_{n}}\left(-\left(a_{n}\right) /\left(2 \sin ^{2} \phi\right)\right)$, we obtain

$$
\begin{aligned}
\operatorname{MGF}_{\beta_{n}}\left(-\frac{a_{n}}{2 \sin ^{2} \phi}\right) \\
=\frac{L m^{m}}{\Gamma(m)} \int_{x=0}^{\infty} e^{-\frac{a_{n} x}{2 \sin ^{2} \phi}} e^{-m x} x^{m-1}[\gamma(m, m x)]^{L-1} d x \\
=\frac{L}{\Gamma(m)}\left(\frac{\sin ^{2} \phi}{\sin ^{2} \phi+\frac{a_{n}}{2 m}}\right) \int_{y=0}^{m} e^{-y} y^{m-1} \\
\times\left[\gamma\left(m, \frac{y \sin ^{2} \phi}{\sin ^{2} \phi+\frac{a_{n}}{2 m}}\right)\right]_{m}^{L-1} d y \\
\approx \\
\quad \frac{L}{\Gamma(m)}\left(\frac{\sin ^{2} \phi}{\sin ^{2} \phi+\frac{a_{n}}{2 m}}\right) \sum_{j=1}^{J} w_{m-1}(j) \\
\times\left[\gamma\left(m, \frac{x_{m-1}(j) \sin ^{2} \phi}{\sin ^{2} \phi+\frac{a_{n}}{2 m}}\right)\right]^{L-1} .
\end{aligned}
$$

In the above equation, the second step is arrived at by the change of integration variable from $x$ to $y$ through $y=x(m+$ $\left.\left(a_{n}\right) /\left(2 \sin ^{2} \phi\right)\right)$, and the third step is due to the application of (11).

Using (7) and (11), we obtain the following simple and accurate numerical approximation for $P_{2}^{\mathrm{SC}}(d)$ :

$$
\begin{aligned}
& P_{2}^{\mathrm{SC}}(d) \frac{1}{\pi} \int_{\phi=0}^{\frac{\pi}{2}} d \phi \prod_{n=1}^{d} \operatorname{MGF}_{\beta_{n}}\left(-\frac{a_{n}}{2 \sin ^{2} \phi}\right) \\
& \approx \frac{1}{2 P} \sum_{k=1}^{P} \prod_{n=1}^{d} \operatorname{MGF}_{\beta_{n}}\left(-\frac{a_{n}}{1-\cos \frac{(2 k-1) \pi}{2 P}}\right) \\
& \approx \frac{1}{2 P}\left[\frac{L}{\Gamma(m)}\right]^{d} \sum_{k=1}^{P} \prod_{n=1}^{d}\left\{\left(\frac{\sin ^{2} \frac{(2 k-1) \pi}{4 P}}{\sin ^{2} \frac{(2 k-1) \pi}{4 P}+\frac{a_{n}}{2 m}}\right)^{m}\right. \\
&\left.\quad \times \sum_{j=1}^{J} w_{m-1}(j)\left[\gamma\left(m, \frac{x_{m-1}(j) \sin ^{2} \frac{(2 k-1) \pi}{4 P}}{\sin ^{2} \frac{(2 k-1) \pi}{4 P}+\frac{a_{n}}{2 m}}\right)\right]^{L-1}\right\} .
\end{aligned}
$$

In the above equation, the second step is arrived at by using the GCQ rule [18], and the last step is due to the substitution of (12).

\section{Cutoff Rate With Selection Diversity}

In this section, we derive the computational cutoff rate $R_{0}$ for a coded communication system with coherent detection on Nakagami fading channels with $L$-path SC diversity. First, we introduce some notation. Let $\mathbf{X}=\left(X_{1}, \ldots, X_{N}\right)$ denote an $N$-length vector of complex-valued $M$-PSK code symbols. The $L N$-length received code-symbol vector is denoted by $\mathbf{r}=\left(r_{1}^{(1)}, \ldots, r_{1}^{(L)}, \ldots, r_{N}^{(1)}, \ldots, r_{N}^{(L)}\right)$, and the corresponding $L N$-length complex fade vector is denoted by $\mathbf{A}_{\alpha}=\left(\alpha_{1}^{(1)} e^{j \theta_{1}^{(1)}}\right.$, $\left.\ldots, \alpha_{1}^{(L)} e^{j \theta_{1}^{(L)}}, \ldots, \alpha_{N}^{(1)} e^{j \theta_{N}^{(1)}}, \ldots, \alpha_{N}^{(L)} e^{j \theta_{N}^{(L)}}\right)$. The $L$-length vector of received symbols and the fade random variables, corresponding to the $i$ th code symbol $X_{i}$, are denoted by $\underline{r}_{i}=\left(r_{i}^{(1)}, \ldots, r_{i}^{(L)}\right)$, and $\underline{\alpha}_{i}=\left(\alpha_{i}^{(1)} e^{j \theta_{i}^{(1)}}, \ldots, \alpha_{i}^{(L)} e^{j \theta_{i}^{(L)}}\right)$, respectively. That is, $\mathbf{r}=\left(\underline{r}_{1}, \ldots, \underline{r}_{N}\right)$ and $\mathbf{A}_{\alpha}=\left(\underline{\alpha}_{1}\right.$, $\left.\ldots, \underline{\alpha}_{N}\right)$. The function $q(\mathbf{X})$ denotes the probability distribution of the codeword $\mathbf{X}$. Finally, an $M$-PSK symbol is denoted by $s_{p}=e^{j 2 \pi p / M}, p=0,1, \ldots, M-1$, and $X_{i} \in\left\{e^{j 2 \pi p / M}\right\}_{p=0}^{M-1}$.

For a discrete $M$-ary input and continuous output channel, with perfect knowledge of the channel state information (CSI) at the receiver, the cutoff rate is defined as [13, pp. 182-183]

$$
\begin{array}{r}
R_{0}=\lim _{N \rightarrow \infty} \max _{q(\mathbf{X})}\left\{-\frac{1}{N} \log _{2}\left(\int_{\mathcal{C}^{N L}} \int_{\mathcal{C}^{N L}}\right.\right. \\
\left.\left.\left[\sum_{\mathbf{X}} q(\mathbf{X}) \sqrt{p\left(\mathbf{r}, \mathbf{A}_{\alpha} \mid \mathbf{X}\right)}\right]^{2} d \mathbf{r} d \mathbf{A}_{\alpha}\right)\right\}
\end{array}
$$

where $p\left(\mathbf{r}, \mathbf{A}_{\alpha} \mid \mathbf{X}\right)$ is the joint pdf of the received sequence $\mathbf{r}$ and the complex fading sequence $\mathbf{A}_{\alpha}$, given the transmitted codeword $\mathbf{X}$, and $\mathcal{C}^{N L}$ is the $L N$-dimensional complex space. The conditional joint pdf, $p\left(\mathbf{r}, \mathbf{A}_{\alpha} \mid \mathbf{X}\right)$, can be written as

$$
p\left(\mathbf{r}, \mathbf{A}_{\alpha} \mid \mathbf{X}\right)=p\left(\mathbf{r} \mid \mathbf{X}, \mathbf{A}_{\alpha}\right) p\left(\mathbf{A}_{\alpha}\right) .
$$

For a symmetric channel, the expression in (14) can be maximized with the equiprobable input distribution

$$
q(\mathbf{X})=\prod_{i=1}^{N} p\left(X_{i}\right)=\frac{1}{M^{N}}
$$

Substituting (16) in (14), we obtain

$$
\begin{aligned}
R_{0}=- & \lim _{N \rightarrow \infty} \frac{1}{N}\left\{\operatorname { l o g } _ { 2 } \left(\int_{\mathcal{C}^{N L}} \int_{\mathcal{C}^{N L}}\right.\right. \\
& {\left.\left.\left[\frac{1}{M^{N}} \sum_{\mathbf{X}} \sqrt{p\left(\mathbf{r} \mid \mathbf{X}, \mathbf{A}_{\alpha}\right)}\right]^{2} d \mathbf{r} p\left(\mathbf{A}_{\alpha}\right) d \mathbf{A}_{\alpha}\right)\right\} . }
\end{aligned}
$$

The expression $\sum_{\mathbf{X}}\left(1 /\left(M^{N}\right) \sqrt{p\left(\mathbf{r} \mid \mathbf{X}, \mathbf{A}_{\alpha}\right)}\right.$ in (17) can be further simplified as follows:

$$
\begin{aligned}
\sum_{\mathbf{X}} \frac{1}{M^{N}} \sqrt{p\left(\mathbf{r} \mid \mathbf{X}, \mathbf{A}_{\alpha}\right)} & =\sum_{\mathbf{X}}\left[\prod_{i=1}^{N} \frac{1}{M} \sqrt{p\left(\underline{r}_{i} \mid X_{i}, \underline{\alpha}_{i}\right)}\right] \\
& =\prod_{i=1}^{N}\left[\sum_{p=0}^{M-1} \frac{1}{M} \sqrt{p\left(\underline{r}_{i} \mid s_{p}, \underline{\alpha}_{i}\right)}\right] \\
& =\prod_{i=1}^{N} \mathcal{T}(i)
\end{aligned}
$$


where $\mathcal{T}(i)=\sum_{p=0}^{M-1}(1 / M) \sqrt{p\left(\underline{r}_{i} \mid s_{p}, \underline{\alpha}_{i}\right)}$. Substituting (18) in (17), we obtain

$$
\begin{aligned}
R_{0}=- & \lim _{N \rightarrow \infty} \frac{1}{N} \\
& \times \log _{2}\left(\int_{\mathcal{C}^{N L}} \int_{\mathcal{C}^{N L}} \prod_{i=1}^{N} \mathcal{T}^{2}(i) d \mathbf{r} p\left(\mathbf{A}_{\alpha}\right) d \mathbf{A}_{\alpha}\right) .
\end{aligned}
$$

Noting that $p\left(\mathbf{A}_{\alpha}\right)=\prod_{i=1}^{N} p\left(\underline{\alpha}_{i}\right)$, and $\mathcal{T}(1), \ldots, \mathcal{T}(N)$ are i.i.d., (19) can be further simplified as

$$
\begin{aligned}
R_{0}= & -\lim _{N \rightarrow \infty} \frac{1}{N} \log _{2}\left(\left[\int_{\mathcal{C}^{L}} \int_{\mathcal{C}^{L}} \mathcal{T}^{2}(i) d \underline{r}_{i} p\left(\underline{\alpha}_{i}\right) d \underline{\alpha}_{i}\right]^{N}\right) \\
=2 & \log _{2}(M)-\log _{2} \\
& \times\left(\sum_{p=0}^{M-1} \sum_{n=0}^{M-1} \int_{\mathcal{C}^{L}} \int_{\mathcal{C}^{L}}\right. \\
& \left.\times \sqrt{p\left(\underline{r}_{i} \mid s_{p}, \underline{\alpha}_{i}\right) p\left(\underline{r}_{i} \mid s_{n}, \underline{\alpha}_{i}\right)} d \underline{r}_{i} p\left(\underline{\alpha}_{i}\right) d \underline{\alpha}_{i}\right) .
\end{aligned}
$$

When $X_{i}=s_{m}$ is the transmitted code symbol, the received symbol, $r_{i}$, at time $i$, at the output of the selection combiner is given by (5). With this, it is not difficult to show that $[1, \mathrm{pp}$. 411-412]

$$
\begin{aligned}
\int_{\mathcal{C}^{L}} \sqrt{p\left(\underline{r}_{i} \mid s_{p}, \underline{\alpha}_{i}\right) p\left(\underline{r}_{i} \mid s_{n}, \underline{\alpha}_{i}\right)} d \underline{r}_{i} & \\
= & \exp \left(-\frac{\beta_{i} E_{s}\left|s_{p}-s_{n}\right|^{2}}{4 N_{0}}\right)
\end{aligned}
$$

where $\beta_{i}=\max \left(\left[\alpha_{i}^{(1)}\right]^{2}, \ldots,\left[\alpha_{i}^{(L)}\right]^{2}\right)$ as given in (4). Substituting (21) in (20), and noting that $\beta_{1}, \ldots, \beta_{N}$ are i.i.d., the cutoff rate $R_{0}$ can be conveniently written as

$$
\begin{aligned}
& R_{0}=2 \log _{2}(M)- \\
& \log _{2}\left(\sum_{p=0}^{M-1} \sum_{n=0}^{M-1} \operatorname{MGF}_{\beta}\left(-\frac{E_{s}\left|s_{p}-s_{n}\right|^{2}}{4 N_{0}}\right)\right) .
\end{aligned}
$$

Notice that the cutoff rate expression in the above equation is a simple-to-compute function of the MGF of the code symbol SNR at the output of the selection diversity combiner. The $R_{0}$ in (22) can be evaluated efficiently either using the exact expression of (10) or an equivalent GLQ approximation of (12). As a quick check, for binary phase-shift keying (BPSK) signals (i.e., $M=2$ ) on an AWGN channel (i.e., $f_{\beta}(x)=\delta(x-1)$ ), we obtain $\operatorname{MGF}_{\beta}\left(-\left(E_{s}\left|s_{p}-s_{n}\right|^{2}\right) /\left(4 N_{0}\right)\right)=e^{-\left(E_{s}\left|s_{p}-s_{n}\right|^{2}\right) /\left(4 N_{0}\right)}$ and the cutoff rate $R_{0}=1-\log _{2}\left(1+e^{-\left(E_{s}\right) /\left(N_{0}\right)}\right)$, in agreement with [1, eq. (7-2-20)]. We also notice that after extracting a factor of $\log _{2}(M)$ from (22), the resulting expression is similar to $[19$, eq. (12.14)].

We evaluate the cutoff rate expression derived in (22) for Nakagami- $m, m \in\{0.5,5\}$, fading channels for various orders of diversity, $L \in\{1,2,3\}$, and for different $M$-PSK constellations, $M \in\{2,4,8\}$. In this paper, in all the numerical and simulation results, the symbol energy per path, $E_{s}$, is scaled by the average value of $\beta, E(\beta)$, so that the average received SNR at the output of the selection combiner is the same, irrespective

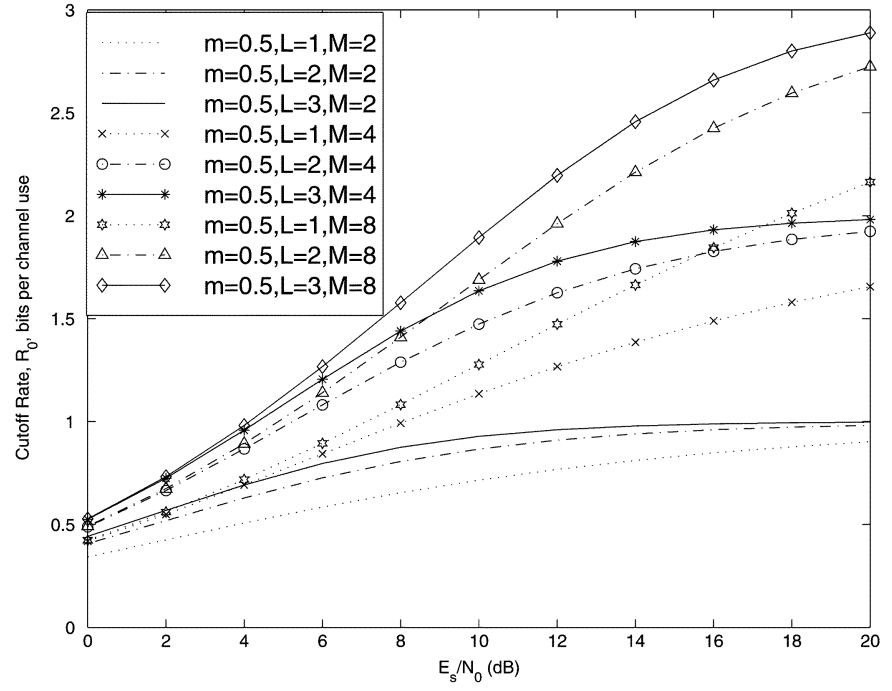

Fig. 1. Computational cutoff rate $R_{0}$, for Nakagami- $m$ fading channels with $L$-antenna SC diversity. $M$ is the constellation size of PSK signal set. $m=0.5$.

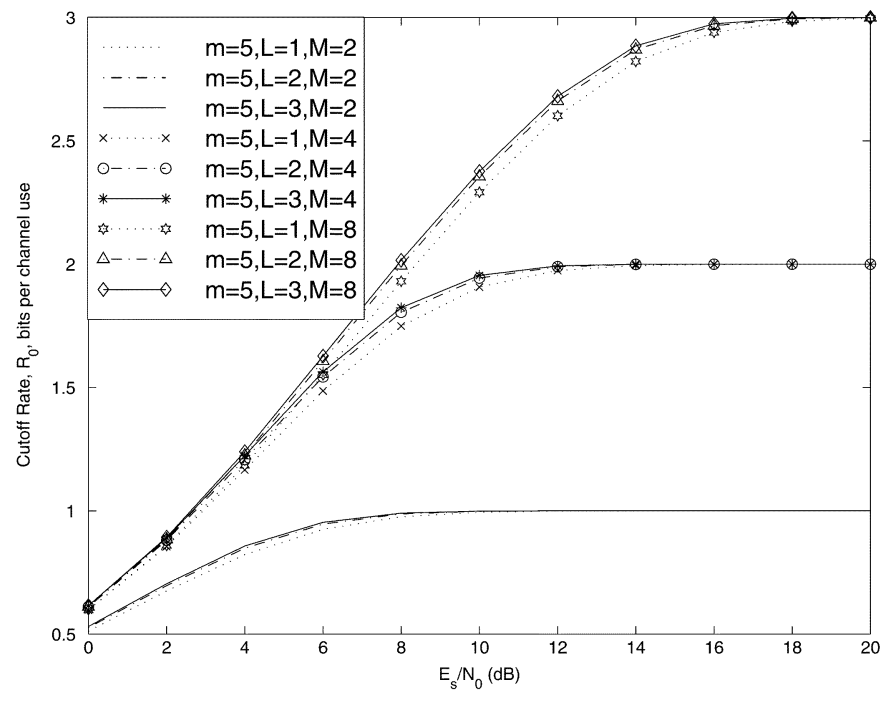

Fig. 2. Computational cutoff rate $R_{0}$, for Nakagami- $m$ fading channels with $L$-antenna SC diversity. $M$ is the constellation size of PSK signal set. $m=5$.

of the Nakagami parameter $m$ and the diversity order $L$, which allows a fair comparison of the performances with various combinations of $m$ and $L$. The cutoff rate plots for various values of $m, L$, and $M$ are shown in Figs. 1 and 2. From these figures, we observe that: 1 ) the cutoff rate is significantly affected when the fading severity index, $m$, is at its lowest value (i.e., for $m=0.5) ; 2)$ there is a diminishing-returns effect on $R_{0}$ as the number of diversity paths, $L$, is increased beyond two; and 3 ) for benign (light fading, large $m$ ) channels, diversity has a minimal effect on the cutoff rate.

\section{BEP BOUNDS FOR TCM AND TURBO CODES}

In this section, using the exact expression for $\operatorname{MGF}_{\beta_{k}}(s)$ (10), and GCQ/GLQ-based approximation for the PEP (13), we present union-bound-based BEP performance for both TCM and turbo codes. 


\section{A. Union Bound on BEP: TCM}

An upper bound on the BEP of a TCM scheme, using the transfer-function bounding technique [10], can be written as [2], [12]

$$
\begin{aligned}
P_{b} \leq & \frac{1}{k \pi} \int_{\theta=0}^{\frac{\pi}{2}}\left\{\sum_{\mathbf{X} \neq \mathbf{Y}} a(\mathbf{X} \rightarrow \mathbf{Y}) \prod_{i \in \mathcal{P}} E_{\beta_{i}}\left[D(\theta)^{\beta_{i}\left|X_{i}-Y_{i}\right|^{2}}\right]\right\} d \theta \\
= & \frac{1}{k \pi} \int_{\theta=0}^{\frac{\pi}{2}}\left\{\sum_{\mathbf{X} \neq \mathbf{Y}} a(\mathbf{X} \rightarrow \mathbf{Y}) \prod_{i \in \mathcal{P}} \mathrm{MGF}_{\beta_{i}}\right. \\
& \left.\times\left(-\frac{E_{s}\left|X_{i}-Y_{i}\right|^{2}}{4 N_{0} \sin ^{2} \theta}\right)\right\} d \theta \\
= & \left.\frac{1}{k \pi} \int_{\theta=0}^{\frac{\pi}{2}} \frac{d}{d I} T(\overline{D(\theta)}, I)\right|_{I=1} d \theta
\end{aligned}
$$

where $k$ is the number of information bits per encoding interval, $\mathbf{X} \rightarrow \mathbf{Y}$ is the event that the transmitted codeword $\mathbf{X}$ is incorrectly decoded as the codeword $\mathbf{Y}, a(\mathbf{X} \rightarrow \mathbf{Y})$ is the number of information bit errors due to this event, $D(\theta)=$ $e^{-\left(E_{s}\right) /\left(4 N_{0} \sin ^{2} \theta\right)}, \mathcal{P}$ is the set of all $p$ such that $X_{p} \neq Y_{p}$, and $T(\overline{D(\theta)}, I)$ is the transfer function of the underlying trellis code where each branch gain is replaced by $E_{\beta}\left[D^{\beta\left|X_{n}-Y_{n}\right|^{2}}(\theta)\right]$.

We evaluate the BEP bound for a 2-state, rate-1/2 encoded TCM scheme on Nakagami fading channels with $m=0.5$ and 5 , and the number of paths $L \in\{1,2,3\}$. The encoder and the signal set mapping are same as that of [8] with the transfer function

$$
\begin{aligned}
T(\overline{D(\theta)}, I) & =\frac{I E_{\beta}\left[D^{2 \beta}(\theta)\right] \times E_{\beta}\left[D^{4 \beta}(\theta)\right]}{1-I E_{\beta}\left[D^{2 \beta}(\theta)\right]} \\
& =\frac{I \mathrm{MGF}_{\beta}\left(-\frac{E_{s}}{2 N_{0} \sin ^{2} \theta}\right) \mathrm{MGF}_{\beta}\left(-\frac{E_{s}}{N_{0} \sin ^{2} \theta}\right)}{1-I \mathrm{MGF}_{\beta}\left(-\frac{E_{s}}{2 N_{0} \sin ^{2} \theta}\right)} .
\end{aligned}
$$

Figs. 3 and 4 show the union bound on the BEP for $m=0.5$ and 5, respectively. Simulation results are also plotted to evaluate the accuracy of the bound on BEP. From Figs. 3 and 4, we see that the bounds are increasingly tight for moderate-to-high SNRs. We also note that, at an error rate of $1 \times 10^{-3}$, more than $2-\mathrm{dB}$ improvement in the performance can be achieved with an additional antenna (from $L=2$ to $L=3$ ) for $m=0.5$, whereas this improvement becomes less than $0.5 \mathrm{~dB}$ for $m=5$. This is due to the fact that $m=5$ corresponds to light fading, and diversity has minimal effect on this channel.

\section{B. Union Bound on BEP: Turbo Codes}

In [20], a transfer-function-based union upper bound is obtained for turbo codes on an AWGN channel. Later, in [9], the same approach is extended for Rayleigh fading channels. In this section, using the approach of [9], we provide upper bounds

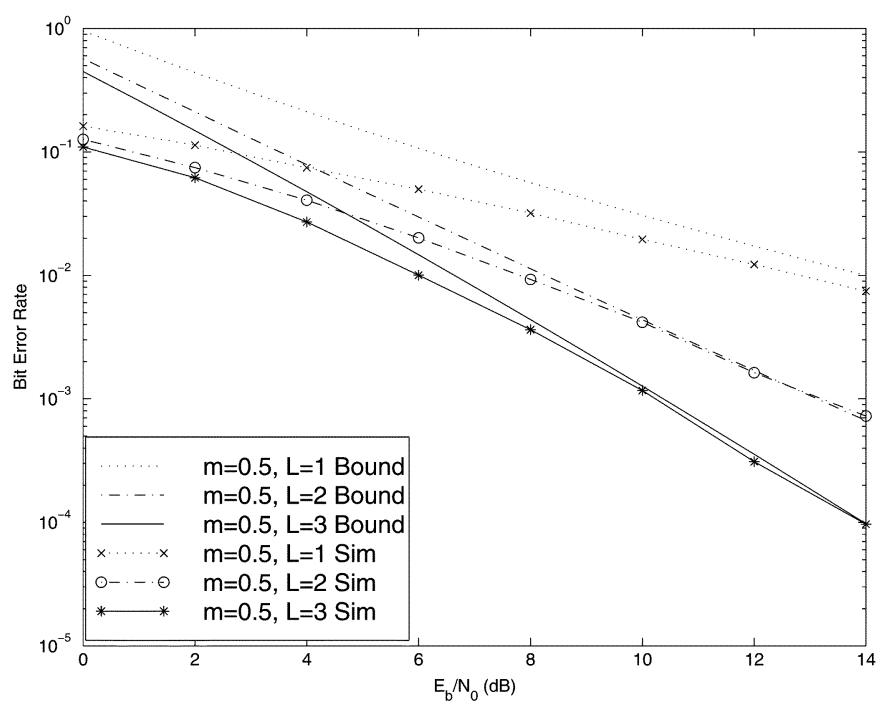

Fig. 3. Bit-error performance of TCM scheme on Nakagami- $m$ i.i.d. fading channel with SC diversity. Number of antennas, $L=1,2,3$, and $m=0.5$. Code rate is $1 / 2$, and the number of encoder states is two.

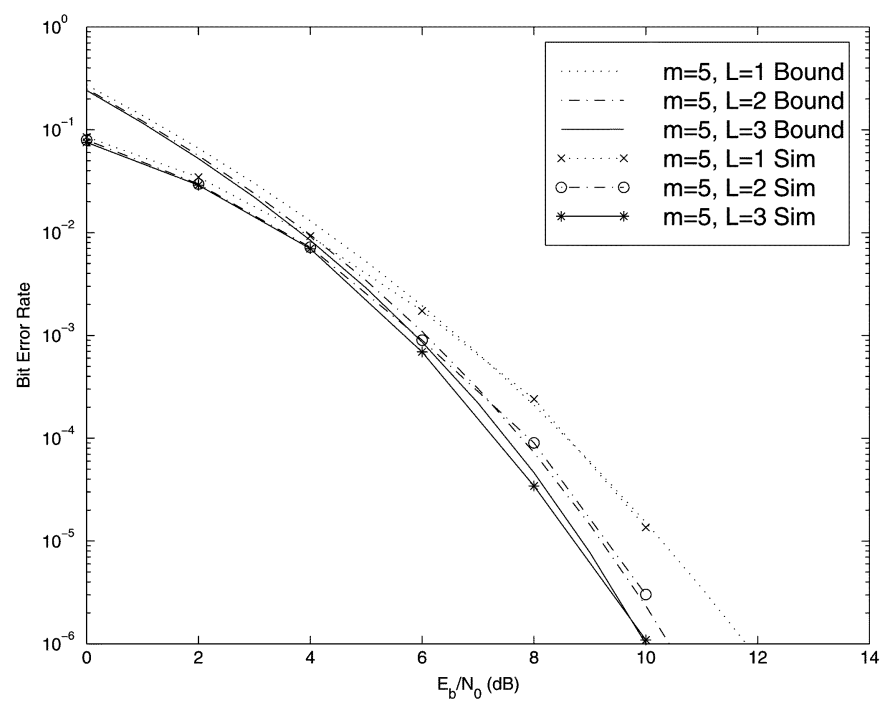

Fig. 4. Bit-error performance of TCM scheme on Nakagami- $m$ i.i.d. fading channel with SC diversity. Number of antennas, $L=1,2,3$, and $m=5$. Code rate is $1 / 2$, and the number of encoder states is two.

on the BEP of turbo codes on Nakagami fading channels with SC diversity. We assume an $(N, K)$ parallel concatenated turbo code with a uniform interleaver, and with a minimum distance of $d_{\min }$. If we denote

$$
t(d)=\sum_{i=1}^{K}(i / K)\left(\begin{array}{c}
K \\
i
\end{array}\right) p(d \mid i)
$$

as the distance spectra of the turbo code, where $p(d \mid i)$ is the conditional probability of the codeword weight being $d$, given the information weight $i$, an upper bound on the BEP can be written as [9]

$$
\overline{P_{b}} \leq \sum_{d=d_{\min }}^{N} t(d) P_{2}(d) .
$$




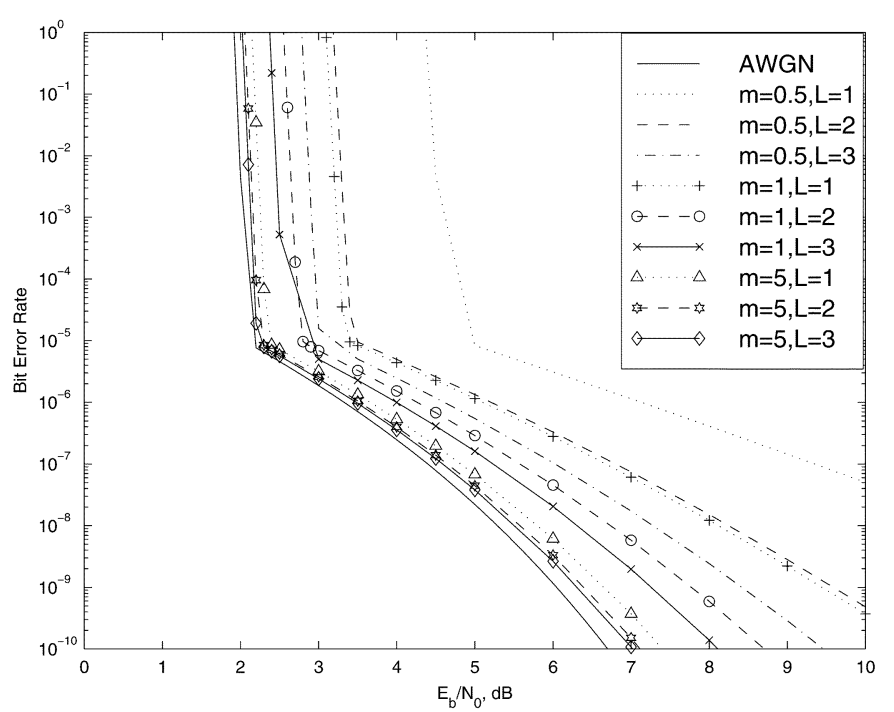

Fig. 5. BEP bounds for turbo codes on interleaved Nakagami- $m$ fading channels with $L$-antenna SC diversity. Information block length is 500 bits with uniform turbo interleaver and code rate $1 / 3$.

TABLE I

$E_{b} / N_{0}$ (IN dB) AT Which the BEP of RATE-1/3 TuRbo Code Diverges on NAKAGAMI- $m$ FADING ChanNel With $L$-PATH SC Diversity. The CORRESPONDING $E_{b} / N_{0}$ REQUIRED TO ACHIEVE CUTOFF RATE $R_{0}$ OF 1/3 ARE Also GIVEN

\begin{tabular}{c|c|c|c|c|c|c}
\hline & \multicolumn{2}{|c|}{$E_{b} / N_{0}, \mathrm{~dB}(m=0.5)$} & \multicolumn{2}{c|}{$E_{b} / N_{0}, \mathrm{~dB}(m=1)$} & \multicolumn{2}{c}{$E_{b} / N_{0}, \mathrm{~dB},(m=5)$} \\
\hline$L$ & $R_{0}$ & Turbo code & $R_{0}$ & Turbo code & $R_{0}$ & Turbo code \\
\hline \hline 1 & 3.7712 & 4.4 & 2.5712 & 3.0 & 1.7712 & 2.1 \\
\hline 2 & 2.6712 & 3.1 & 2.0712 & 2.5 & 1.6712 & 2.0 \\
\hline 3 & 2.3712 & 2.7 & 1.9712 & 2.3 & 1.5712 & 2.0 \\
\hline \hline
\end{tabular}

In Fig. 5, we plot the union bound on BEP for a rate-1/3 turbo code, whose constituent encoders' generator polynomials are $(1,21 / 37)_{8}$, and the encoders are separated by a uniform interleaver. The bound is evaluated for Nakagami- $m$ fading channels, $m \in\{0.5,1,5\}$, with $L$-path SC diversity, $L \in\{1,2,3\}$, and for an input block length of 500 information bits (i.e., 1500 code symbols). Also shown in Fig. 5 is the bound for the AWGN channel (i.e., $m \rightarrow \infty$ and $L=1$ ). An important observation to make in Fig. 5 is that the union bound on BEP diverges at low $E_{b} / N_{0}$ values and renders the analytical results ineffective in this SNR range. In [20], it is reported that for the $E_{b} / N_{0}$ values less than the computational cutoff rate values, turbo code BEP bounds exhibit divergence behavior. We would like to point out that such a phenomenon is observed for Nakagami- $m$ fading with $L$-path SC diversity, as well. To do this, we calculate the minimum $E_{b} / N_{0}$ required to achieve a cutoff rate of $1 / 3$ with BPSK signals, and the $E_{b} / N_{0}$ at which our rate- $1 / 3$ turbo-code BEP bound diverges. These two SNR values are given in Table I for various values of $m$ and $L$ for a block length of 500 information bits. From Table I, we observe the following. First, the union bound on BEP for turbo codes with SC diversity on fading channels diverges at $E_{b} / N_{0}$ values below the SNR required to achieve the computational cutoff rate, thus establishing similar observations made in [20]. Second, for small orders of diversity $(L)$ and for severe fading (small $\mathrm{m}$ ), the difference between the $E_{b} / N_{0}$ at which the BEP bound diverges and that which is required to achieve $R_{0}=1 / 3$ is high, relative to what it is when we have large $L$ and/or large $m$.

\section{CONCLUSION}

We derived some analytical tools for the performance analysis of coded coherent communication systems on i.i.d. Nakagami- $m$ fading channels with SC diversity. First, we obtained an exact expression for the MGF of the SNR of a code symbol at the output of the selection combiner. Next, we proposed a simple, yet accurate, numerical solution, based on GCQ and GLQ rules, for the PEP of coded $M$-PSK signals on Nakagami- $m$ fading channels with $L$-path SC diversity. Using the PEP expressions, we presented union bounds on the bit-error performance of TCM and turbo codes. Finally, we derived an exact expression for the channel cutoff rate for the aforementioned coded system, as a function of the MGF of the SNR at the output of the combiner.

\section{REFERENCES}

[1] J. G. Proakis, Digital Communications. New York: McGraw-Hill, 1995.

[2] M. K. Simon and M. S. Alouini, Digital Communications Over Generalized Fading Channels: A Unified Approach to the Performance Analysis. New York: Wiley, 2000.

[3] G. Ungerboeck, "Trellis-coded modulation with redundant signal setsPart I: Introduction,” IEEE Commun. Mag., vol. 25, pp. 5-11, Feb. 1987.

[4] D. Divsalar and M. K. Simon, "The design of trellis-coded MPSK for fading channels: Performance criteria," IEEE Trans. Commun., vol. 36, pp. 1004-1012, Sept. 1988.

[5] C. Berrou, A. Glavieux, and P. Thitimajshima, "Near-Shannon-limit error-correcting coding and decoding: Turbo codes," in Proc. IEEE Int. Conf. Communications, 1993, pp. 1064-1070.

[6] H. Holma and A. Toskala, Eds., WCDMA for UMTS: Radio Access for Third Generation Mobile Communications. New York: Wiley, 2001.

[7] R. G. McKay, P. J. McLane, and E. Biglieri, "Error bounds for trellis coded MPSK on a fading mobile satellite channel," IEEE Trans. Commun., vol. 39, pp. 1750-1761, Dec. 1991.

[8] W.-C. Lin and Y. T. Su, "Bit error bounds for trellis-coded MPSK in mixed fading channels," IEEE Trans. Veh. Technol., vol. 46, pp. 900-909, Nov. 1997.

[9] E. K. Hall and S. G. Wilson, "Design and analysis of turbo codes on Rayleigh fading channels," IEEE J. Select. Areas Commun., vol. 16, pp. 160-174, Feb. 1998.

[10] A. J. Viterbi and J. K. Omura, Principles of Digital Communication and Coding. New York: McGraw-Hill, 1979.

[11] J. K. Cavers and P. Ho, "Analysis of the error performance of trelliscoded modulation in Rayleigh fading channels," IEEE Trans. Commun., vol. 40, pp. 74-80, Jan. 1992.

[12] C. Tellambura, "Evaluation of the exact union bound for trellis-coded modulations over fading channels," IEEE Trans. Commun., vol. 46, pp. 1693-1699, Dec. 1996.

[13] R. Gallager, Information Theory and Reliable Communication. New York: Wiley, 1968.

[14] S. A. Al-Semari and T. E. Fuja, "Performance analysis of coherent TCM systems with diversity reception in slow Rayleigh fading," IEEE Trans. Veh. Technol., vol. 48, pp. 198-212, Jan. 1999.

[15] I. S. Gradshteyn and I. M. Ryzhik, Table of Integrals, Series and Products, 5th ed. San Diego, CA: Academic, 1994.

[16] A. Ramesh, A. Chockalingam, and L. B. Milstein, "Bounds on the performance of turbo codes on Nakagami fading channels with diversity combining," in Proc. IEEE GLOBECOM, Nov. 2001, pp. 1199-1204.

[17] J. W. Craig, "A new simple and exact result for calculating the probability of error for two-dimensional signal constellations," in Proc. IEEE MILCOM, Oct. 1991, pp. 571-575.

[18] W. H. Press, S. A. Teukolsky, W. A. Vetterling, and B. P. Flannery, Numerical Recipies in C: The Art of Scientific Computing. Cambridge, U.K.: Cambridge Univ. Press, 1992.

[19] S. Benedetto and E. Biglieri, Principles of Digital Transmission with Wireless Applications. Boston and New York: Kluwer Academic/ Plenum, 1999.

[20] D. Divsalar, S. Dolinar, R. J. McEliece, and F. Pollara, "Transfer Function Bounds on the Performance of Turbo Codes," Jet Propulsion Lab., Calif. Inst. Technol., Pasadena, CA, TDA Progress Rep. 42-122, Aug. 1995. 


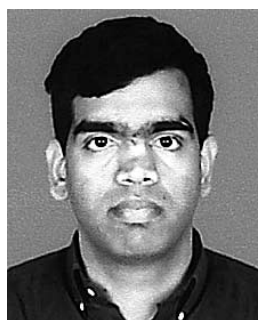

Ramesh Annavajjala (M'02-S'03) received the B.Tech. degree in electronics and communication engineering from the National Institute of Technology (N.I.T), Warangal, India, in May 1998, and the Masters degree in telecommunications from the Indian Institute of Science, Bangalore, India, in January 2001. Currently, he is working toward the $\mathrm{Ph} . \mathrm{D}$. degree at the University of California, San Diego.

From July 1998 to July 1999, he was with CMC R \& D Center, Hyderabad, India, and from February 2001 to August 2002, he was a Systems Design Engineer at Synopsys Inc., Bangalore, India, in the Wireless and Broadband Communications Group. His research interests lie in the areas of wireless communications, design and analysis of multiple-antenna systems, and wideband CDMA systems.

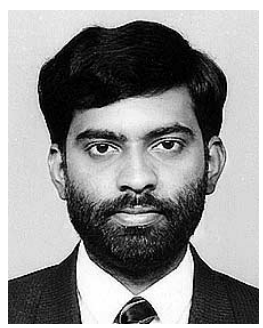

A. Chockalingam (S'92-M'93-SM'98) received the B.E. (Honors) degree in electronics and communication engineering from the P.S.G. College of Technology, Coimbatore, India, in 1984, the M.Tech. degree with specialization in satellite communications from the Indian Institute of Technology, Kharagpur, India, in 1985, and the Ph.D. degree in electrical communication engineering (ECE) from the Indian Institute of Science (IISc), Bangalore, India, in 1993.

During 1986-1993, he worked with the Transmission R \& D Division, Indian Telephone Industries Ltd., Bangalore, India. From December 1993 to May 1996, he was a Postdoctoral Fellow and an Assistant Project Scientist at the Department of Electrical and Computer Engineering, University of California, San Diego (UCSD). From May 1996 to December 1998, he was with Qualcomm, Inc., San Diego, CA, as a Staff Engineer/Manager in the Systems Engineering Group. In December 1998, he joined the faculty of the Department of ECE, IISc, Bangalore, India, where he is an Associate Professor, working in the area of wireless communications. He was a Visiting faculty to UCSD during summers of 1999-2002.

Dr. Chockalingam is a recipient of the Swarnajayanti Fellowship from the Department of Science and Technology, Government of India. He is an Associate Editor of the IEEE TRANSACTIONS ON VEHICULAR TECHNOLOGY.

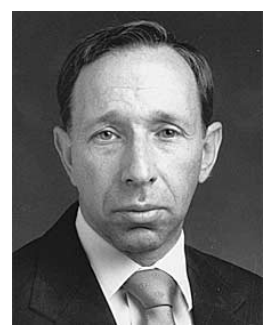

Laurence B. Milstein (S'66-M'68-SM'77-F'85) received the B.E.E. degree from the City College of New York, New York, NY, in 1964, and the M.S. and $\mathrm{Ph} . \mathrm{D}$. degrees in electrical engineering from the Polytechnic Institute of Brooklyn, Brooklyn, NY, in 1966 and 1968, respectively.

From 1968 to 1974, he was with the Space and Communications Group of Hughes Aircraft Company, and from 1974 to 1976 , he was a member of the Department of Electrical and Systems Engineering, Rensselaer Polytechnic Institute, Troy, NY. Since 1976, he has been with the Department of Electrical and Computer Engineering, University of California at San Diego, La Jolla, where he is a Professor and former Department Chairman, working in the area of digital communication theory with special emphasis on spread-spectrum communication systems. He has also been a consultant to both government and industry in the areas of radar and communications.

Dr. Milstein was an Associate Editor for Communication Theory for the IEEE TRANSACTIONS ON COMMUNICATIONS, an Associate Editor for Book Reviews for the IEEE TRANSACTIONS ON INFORMATION THEORY, an Associate Technical Editor for the IEEE Communications Magazine, and the Editor-in-Chief of the IEEE Journal on SELECTEd AREAS IN COMMUNiCATIONS. He was the Vice President for Technical Affairs in 1990 and 1991 of the IEEE Communications Society, and has been a member of the Board of Governors of both the IEEE Communications Society and the IEEE Information Theory Society. He is a former Chair of the IEEE Fellows Selection Committee, and a former Chair of ComSoc's Strategic Planning Committee. He is a recipient of the 1998 Military Communications Conference Long Term Technical Achievement Award, an Academic Senate 1999 UCSD Distinguished Teaching Award, an IEEE Third Millenium Medal in 2000, the 2000 IEEE Communication Society Armstrong Technical Achievement Award, and the 2002 MILCOM Fred Ellersick Award. 